\title{
A WSN-based Health and Tracking System for Green Cyclist Community
}

\author{
Haw-Yun Shin and I-Hsiang Chen \\ Department of Computer Science and Engineering \\ National Taiwan Ocean University \\ Keelung, Taiwan \\ hyshin@ntou.edu.tw
}

\author{
Chung-Ming Own \\ School of Computer Software \\ Tianjin University \\ Tianjin, China \\ chungming.own@tju.edu.cn
}

\begin{abstract}
We used the green power and WSN techniques to address issues related to cycling activities, including health records, trajectory monitoring, group positioning, and immediate safety issues. Each bicycle is equipped with a green power module, a unique WSN identification device and an optional biomedical monitoring device. GPS is not required. Bike paths are equipped with routers and gateways that serve as a bridge between the bicycle devices and the cloud server. The routers collect and transmit the cyclists' unique identification and biomedical data to the cloud server through the gateway. The cloud service platform provides users to monitor and calibrate the cyclists' trajectories, as well as their activity and health records. Furthermore, the cloud service platform includes a path establishment simulation system that enables a path manager to simulate paths before they are physically provided to determine the ideal establishment method for providing router and gateway.
\end{abstract}

Keywords- wireless sensor network; green power; trajectory tracking; group positioning

\section{INTRODUCTION}

Wireless sensor networks (WSNs) possess many advantageous characteristics, such as simple large-scale establishment, low power consumption, stable wireless transmission and installation, the ability to be equipped with various sensors, and broad applications and innovations. For example, for daily lifestyle applications, [1] designed a WSN-based intelligent gas monitoring system. To achieve efficient energy use, Yeh et al. [2] employed the wireless sensor node technique, examined people's habits and changes in their environment, and implemented an energy-saving light system. Chen et al. [3] used RFID and WSN to design a system that provides group travel guides. For medical management, Dilmaghani et al. [4] suggested installing WSNs in the homes of multiple myeloma patients to facilitate real-time monitoring of their physiological signals. [5] recommended employing radio-frequency identification (RFID) combined with WSN in intelligent hospital space platforms to manage drugs and medical equipment, and assist doctors and nurses in monitoring patient conditions. For environmental monitoring, [6] placed sensors on public transportation for effective monitoring and to collect traffic, air quality, and climate data. [7-8] employed WSN techniques to investigate and detect the habitat conditions of animals and birds, analyzing and collecting data at a distance. Chintalapudi et al. [9] suggested using inexpensive wireless sensor nodes to continuously detect building structures to maintain their integrity. Xiaojuan et al. [10] recommended using WSN techniques in oil refineries to construct sensor networks for hydrogen sulfide $(\mathrm{H} 2 \mathrm{~S})$ detection with comprehensive functions.
We used WSN techniques to conduct extensive research and development of various issues related to cycling activities, including green power supply [11], activity and health records [12], trajectory monitoring and group positioning [13], and immediate safety issues [14]. Every bicycle that joined the study community was equipped with a unique WSN identification device. Before departing, users could log into the system service Web site to select their team members. As the user rides along the green bike path, the ZigBee routers along the path continuously collect the users' location and biomedical data. Data of the path's microclimate are also transmitted to the cloud server through the ZigBee gateway [15]. Thus, we can monitor and calibrate the users' trajectories, as well as their activity and health records. We can also transmit essential group messages to the user-end devices. Furthermore, the riders' safety and the anti-theft mechanisms of the bicycles are simultaneously monitored by the system.

As shown in Fig. 1, each bicycle is equipped with green power sources, a unique ZigBee identification module and an optional biomedical monitoring and display device. Bicycles do not require GPS, and when cyclers are cycling on the green paths, routers and gateways on the path collect data of the passing cyclists (bicycle ID and cyclists' biomedical data) and then transmit the data to cloud servers. The cloud service platform is then used to provide services related to group positioning and trajectory monitoring, activity and health records, and immediate safety. The cloud service platform also includes a path establishment system that provides path managers with the ability to supplement or maintain paths. This system enables managers to simulate paths before they are physically established. The establishment procedures are conducted as follows: according to local bicycle traffic and the path shape, managers locate the optimum router quantity and gateway positions for establishment and installation. After simulation, establishment reports regarding the latitude and longitude of all routers and gateways are produced. Construction personnel then construct the paths according to the content of the report. After construction is completed, the MAC addresses of the routers and gateways are input into the server via the administrator page to complete the establishment of wireless sensor nodes on bike paths.

The device design comprises a simple node and a super node. Although both nodes possess the ZigBee wireless transmission function and detection sensors, the super node can process greater volumes of data and has superior computing capabilities. In this study, each bicycle uses a simple node, and the routers and gateways use a super node. The primary differences and appearances between simple and super nodes are shown in Fig. 1. 


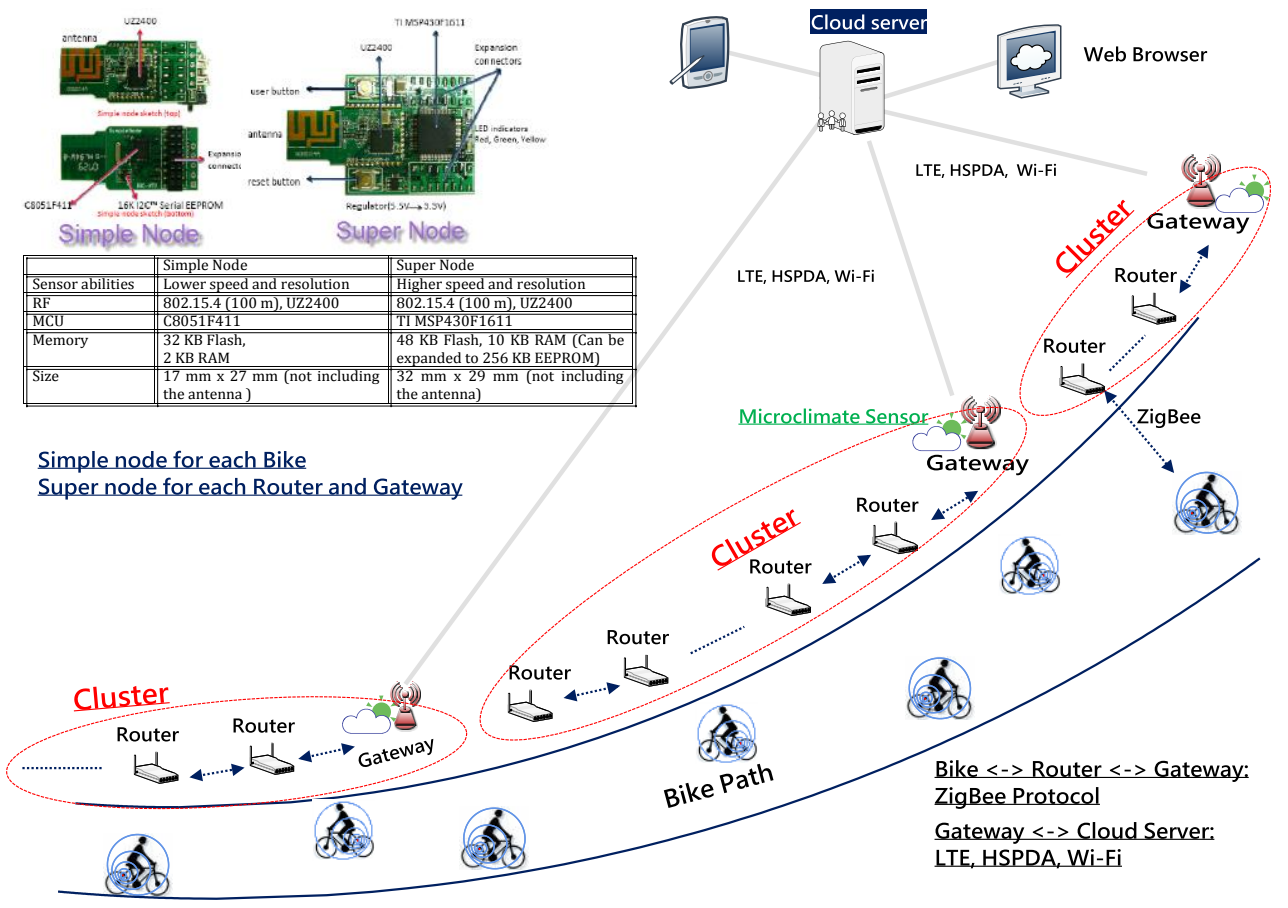

Figure 1. Framework for the green bike path.

\section{FUnCtIONS DESIGNING}

\section{A. Bike path designing}

Bike paths are equipped with two WSN devices (router and gateway) that collect data and serve as a bridge between the bicycle devices and the cloud server. Routers are used to transmit data packets to the ZigBee bicycle device bidirectionally. The routers can also transfer data collected by neighboring routers to the gateway. The stable communication range for routers can be used to determine the installation distance between routers.

One end of the gateway is the ZigBee module (Super node), and the other end is various wireless transmission interfaces, such as HSDPA, Wi-Fi, or LTE. This design provides gateway protocol conversion functions. Besides collecting and exchanging the bicycles' ZigBee data packets, the gateway also forwards the bicycle data collected by routers (controlled by the gateway) to the cloud server. Data transmissions from the cloud server to bicycles must be broadcast by the gateway. During establishment and installation, a number of routers are linked to one gateway to form a cluster. Thus, one bike path comprises a number of clusters. A portion of the gateways possess a microclimate sensor and air-quality monitoring device to obtain climate data, such as the humidity and temperature, UV radiation, wind speed, and air quality, during journeys.

These two path-end platforms do not require GPS because the cloud server establishes the location and geographic coordinates of all of the WSN devices on the path in advance. Because the power consumed by gateway devices is higher, con- structing these devices in an area with a power supply is a priority and independent power supply is only a secondary option. The routers only comprise a ZigBee module, which indicates that their power consumption is low. However, because of the many devices installed, independent power supply is required for these devices. For the various gateway locations without a power supply, we designed wind and light power input devices. Figure 2 shows the microclimate sensor and wind and light power input devices for gateways and routers.

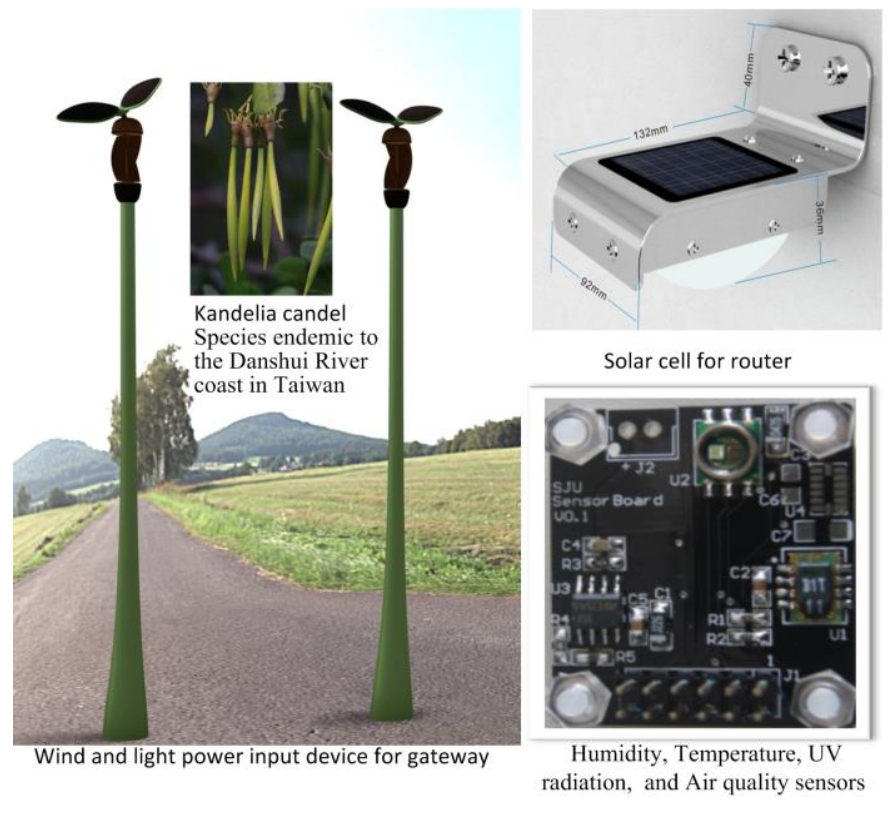

Figure 2. Microclimate sensor and wind and light power input devices for gateways and routers 


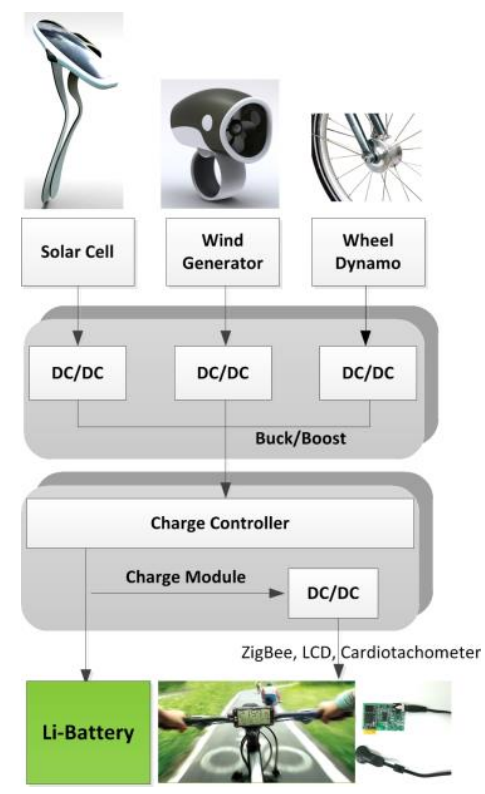

Figure 3. Green power supply module

\section{B. Bike device}

The bike device includes a ZigBee module with a unique identification address. Three additional modules can also be installed, that is, a green power supply module, a biomedical detection device, and an LCD display, as shown in Fig. 3. The functions of the bike device are described below.

- The design of power input device included an electrical energy converter with a wind-light- wheel power system and the development of storage techniques. The design increases the reliability of power devices and provides bicycles with sufficient and self-sustaining power.

- When bicycles pass the gateways or routers established on the path, the ZigBee module transmits the bicycle' $\mathrm{s}$ ID and relevant information of the cyclist to the cloud server.

- $\quad$ By equipping the plug-and-play biomedical detection or sensing module, the bike device can also collect cyclist data, including their heart rate, heart rate variations, temperature, and cycling speeds.

- $\quad$ The bicycles are equipped with emergency buttons that enable the cyclists to send emergency or distress signals through the routers on the path to the cloud servers and other team members for immediate assistance.

- ZigBee chips are built into the bicycles during the manufacturing process; the 64-bit extended address of the chip is used as the bicycle' $s$ unique identification and for anti-theft measures.

\section{Cloud service platform}

The cloud service platform provides the following services:

- Member registration and bicycle community establishment.
- $\quad$ Employs Google Earth to achieve bike path reality preview function.

- Employs Google Maps to enable managers and users to locate specific communities and cyclists using Internet browsers on PCs and PDAs. In addition, the system provides users with requested information through mobile phone short message (SMS).

- $\quad$ Bicycle communities can locate other members, transmit messages, assemble teams, and announce event information.

- If a specific user presses the emergency button on their bicycle, the system can execute the appropriate emergency response; for example, notify other community members, patrol cars, or maintenance trucks.

- $\quad$ The module design includes a path establishment and simulation system to enable managers to develop and maintain the bike paths.

- The servers provide information for searches or enquiries, such as the traffic conditions and air quality and record members' cycling trajectories.

\section{Communications}

To perform the bicycle positioning function, the simple node on the bicycles (termed the bike node) must communicate with routers on the bike path at a fixed time. The router transmits the unique MAC address of the bicycle and the router's ID to the server via a gateway. Thus, the router range in which the bicycle is located is recorded to determine the bicycle location. When cyclists stop and press the emergency button on their bicycle, the bicycle transmits several emergency distress signals to the server via the routers and gateways. The server then broadcasts emergency signals notifying the other team members on the path, or informs paramedics to proceed to the scene. However, for these functions to be performed, the routers and bike nodes must be capable of two-way data transmissions to the server.

\section{A. Transmissions between the bike node and router}

Because of the frequent traffic of bicycles on bike paths, we employed non-beacon strategies (i.e., the broadcast method) for the design of transmissions between bike nodes and the routers on the path. The source address of the packet represents the MAC address of the bicycle ID, enabling system identification and positioning functions. The first byte of the payload is the header, which indicates that this is the packet of the bicycle information system, and the second byte of the payload is the control code, which indicates the message type. Transmissions and communications between bike-nodes and routers do not adopt acknowledgement mechanisms and do not re-send messages when transmissions fail.

\section{B. Transmissions between the router and cloud server}

Routers that employ the data aggregation uploading technique use MAC addresses as a reference for routing. The location of the upper-level router (closer to the gateway) is the router's MAC address plus one, and the location of the lower- 
layer router is the router's MAC address minus one. When a router does not receive packet data from the gateway or neighboring routers, it exists in the data aggregation state. This indicates that the router continuously collects data within the communication range but does not upload data. If the router reaches the upload time, or receives a packet sent by the lower-layer router, the router compiles the data from the buffer zone and the packet data received from the lower-layer router into one packet. This packet is then transmitted to the upper-level router, and the data aggregation buffer is emptied after uploading. The router then recollects bicycle data within the domain. After the gateway receives the packet, it dissembles the aggregated data and uploads it to the server.

\section{Transmissions between the cloud server and bike node}

The volume of messages transmitted between the server and the bike node is not substantial. To initiate communication between the cloud server and the bike node, broadcast techniques can be used to transmit ZigBee packets. Although this method increases the broadcast packets transmitted or circulated between routers, it is the most pragmatic approach. To transmit data from the server to specific bike nodes, the gateway location of the bike node recorded in the server must be obtained. Data is then transmitted to the specified gateway and neighboring gateways, which then broadcast the data to the target bike nodes via the routers controlled by the gateways.

\section{BIKE PATH ESTABLISHMENT AND SIMULATION SYSTEM}

\section{A. Functions of the bike path establishment and simulation system}

The bike path establishment system is situated on the cloud service platform and provides path managers with functions to develop and maintain bike paths. The system can be divided into four modules, that is, Web simulation interface module, path node establishment module, simulator intermediary program module, and simulator module. Each module is described below.

1) Web simulation interface module: Because numerous parameter settings are required for each simulation and when the size of the simulation network increases, more input parameters are required, this module provides a more userfriendly interface. Users are only required to input a few parameters related to the system, such as the number of bicycles, gateway locations, bicycle messaging interval times, and simulation times. This module automatically converts the data into the parameters required by the simulator, and then transmits the parameters to the simulator intermediary program module.

2) Path node establishment module is combined with Google Maps API. Users can use a scaled map to select the locations of routers and gateways, and the module then automatically converts the GPS coordinates for the selected location into the coordinate specifications required for the simulator. Figure 4 shows the locations of routers and gateways established by a user through the Google Maps API. After the path node establishment module obtained the coordinates, we applied the spherical distance equation to determine the relative location of each node. This module also enables users to define transmission ranges; If users establish routers and gateways outside the transmission range, the system displays a warning window informing users that the selected sensor nodes are outside the transmission range.

3) Simulator intermediary program module: After the users have established the sensor node locations and related parameters, the parameters set by the users can be accessed through the simulator intermediary program. These parameters are then converted into the simulator format, transmitted to the simulator module, and simulated. When the simulation is complete, the accessed simulation results are transferred to specific pages to display the simulation data and relevant diagrams.

4) Simulator module: This system employs OMNeT++ 4.2.2[16] and MiXiM[17] as simulators. The primary program for the overall network simulator is conducted on OMNeT++. MiXiM is the unofficially OMNeT++ network module, which includes a more comprehensively developed ZigBee network model. For the system to simulate a ZigBee network topology environment, a ZigBee-related network model must be installed on the OMNeT++ simulator, and the relevant functions provided by MiXiM, such as the ZigBee coordinator, ZigBee router, and ZigBee-end device, must be adjusted. These procedures and functions can then correspond with the gateway, router, and bike node functions employed in this study.

Thus, users can simultaneously conduct path node establishment, path simulation, and simulation result retrieval functions using these four modules. Each group of simulation parameters established by the users is transmitted to the simulator for simulation. After simulation, the simulation results are generated and graphically displayed on a Web page.

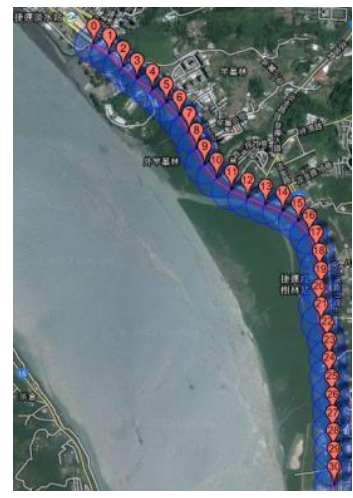

(a)

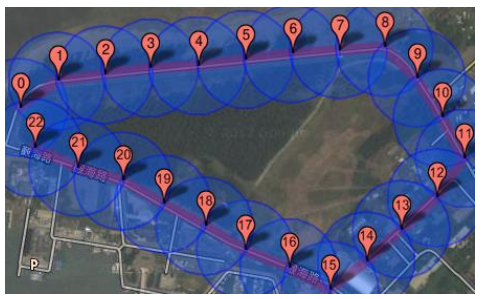

(b)
Figure 4. Path establishment examples. (a) Danshui Golden Waterfront in northern Taiwan. (b) Circular path.

\section{B. Path establishment simulation example}

As shown in Fig. 4, we used the Danshui Golden Waterfront in northern Taiwan and a circular path as our establishment and installation examples, and developed the optimum 
path establishment method under different parameter settings. In these two examples, the sensing range of the routers and gateways was $100 \mathrm{~m}$. The routers and gateways do not broadcast the same messages repeatedly. The bicycles move at speeds ranging from $10 \mathrm{~km} / \mathrm{hr}(2.7 \mathrm{~m} / \mathrm{ses})$ to $20 \mathrm{~km} / \mathrm{hr}$ $(5.5 \mathrm{~m} / \mathrm{sec})$ on the paths. A bicycle moving at a speed of 20 $\mathrm{km} / \mathrm{hr}(5.5 \mathrm{~m} / \mathrm{sec})$ will take approximately $18 \mathrm{sec}$ to travel between nodes. If the bicycle sensor requires $18 \mathrm{sec}$ to transmit messages to the server, when the message is delivered, the bicycle may be by the next router. However, the location of the previous router will be recorded in the server, causing positioning errors.

\begin{tabular}{|l|c|c|c|c|c||}
\hline \hline \multicolumn{5}{|c|}{ Mean } \\
\hline \hline Configulation & $\begin{array}{c}\text { Packet Received } \\
\text { (Pkt/Sec) }\end{array}$ & $\begin{array}{c}\text { Packet Forwarded } \\
\text { (Pkt/Sec) }\end{array}$ & $\begin{array}{c}\text { Delay } \\
\text { (Sec/Pkt) }\end{array}$ & $\begin{array}{c}\text { Dropped } \\
\text { (Pkt) }\end{array}$ & $\begin{array}{c}\text { Success } \\
\text { Rate }\end{array}$ \\
\hline $\mathrm{C}(15,100,3,2)$ & 20.19176 & 19.49407 & 2.70928 & 192.66667 & 0.93432 \\
\hline $\mathrm{C}(5,100,3,2)$ & 22.76935 & 22.36704 & 3.47272 & 242 & 0.9175 \\
\hline $\mathrm{C}(10,100,3,2)$ & 20.781 & 20.13944 & 2.91968 & 199.33333 & 0.93205 \\
\hline $\mathrm{C}(0,100,3,2)$ & 26.88978 & 26.90204 & 3.90147 & 287 & 0.90216 \\
\hline \hline
\end{tabular}

(a)

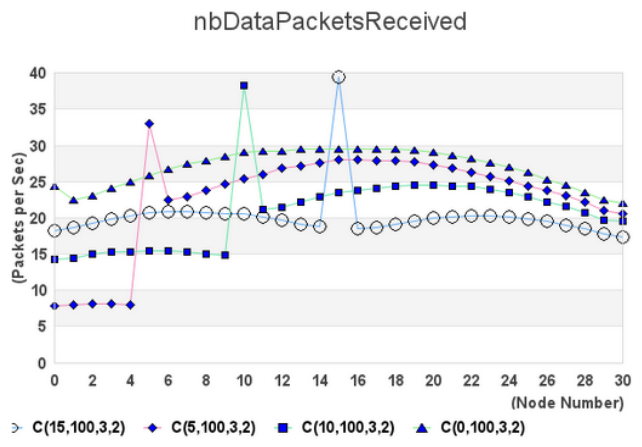

(b)

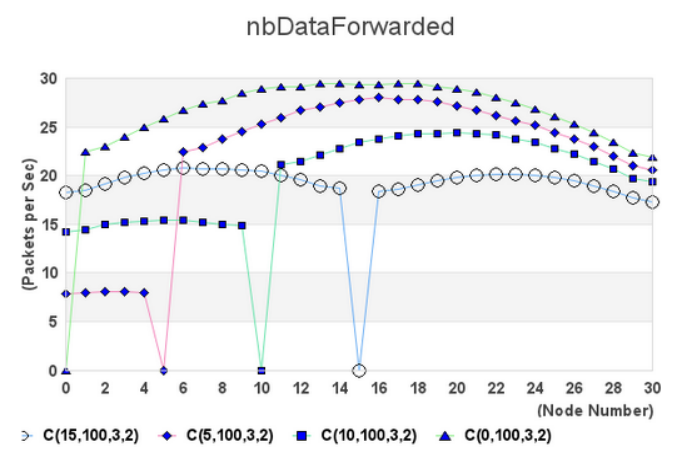

(c)

Figure 5. (a) Performance comparisons for different gateway locations along the Danshui Golden Waterfront (b) The number of packets received by routers. (c) The number of forward by routers.

1) The Danshui Golden Waterfront path: As shown in Fig. 4(a), the length for the bike path employed in this study was approximately $3 \mathrm{~km}$, and a total of 31 routers (including one gateway) were installed. We used $\mathrm{C}(\mathrm{X}, \mathrm{Y}, \mathrm{Z}, \mathrm{A})$ to represent the simulation parameters, where $\mathrm{X}$ represents the serial number of the gateway locations on the path, $\mathrm{Y}$ represents the number of bicycles, $\mathrm{Z}$ represents the simulation time, and $\mathrm{A}$ represents the bicycle messaging interval time. The simulation results show that the closer the gateway position is to the center of a straight path, the better the gateway performance. According to Fig. 5(b) and 5(c), when the gateways were located in various positions, the number of packets received and forwarded by each router was, from the highest to lowest, $\mathrm{C}(0,100,3,2), \mathrm{C}(5,100,3,2), \mathrm{C}(10,100,3,2)$, and $\mathrm{C}(15,100,3,2)$. As the gateway location approaches the center of the path, the number of packets received and forwarded by each router declines, indicating a smaller router load. Figure 5(a) shows the data for four gateway location configurations. By comparing the simulation results for five performance factors (the mean number of packets received, the mean number of packets forwarded, the mean packet delay, and the mean number of packets dropped, as well as the mean packet transmission success rate), we found that of the four simulation configurations, $\mathrm{C}(15,100,3,2)$ had the lowest number of packets dropped, and the highest success rate.

\begin{tabular}{||c|c|c|c|c|c|}
\hline \hline \multicolumn{7}{|c|}{ Mean } \\
\hline \hline Configulation & $\begin{array}{c}\text { Packet Received } \\
\text { (Pkt/Sec) }\end{array}$ & $\begin{array}{c}\text { Packet Forwarded } \\
\text { (Pkt/Sec) }\end{array}$ & $\begin{array}{c}\text { Delay } \\
(\text { Sec/Pkt) }\end{array}$ & $\begin{array}{c}\text { Dropped } \\
\text { (Pkt) }\end{array}$ & $\begin{array}{c}\text { Success } \\
\text { Rate }\end{array}$ \\
\hline $\mathrm{C}(15,100,3,2)$ & 30.59758 & 29.42702 & 3.27687 & 403 & 0.86261 \\
\hline $\mathrm{C}(11,100,3,2)$ & 30.54469 & 29.36616 & 3.2582 & 398.66667 & 0.86409 \\
\hline $\mathrm{C}(5,100,3,2)$ & 30.41111 & 29.28662 & 3.27396 & 395 & 0.86534 \\
\hline $\mathrm{C}(0,100,3,2)$ & 30.88768 & 29.83561 & 3.21242 & 331.33333 & 0.88705 \\
\hline \hline
\end{tabular}

(a)

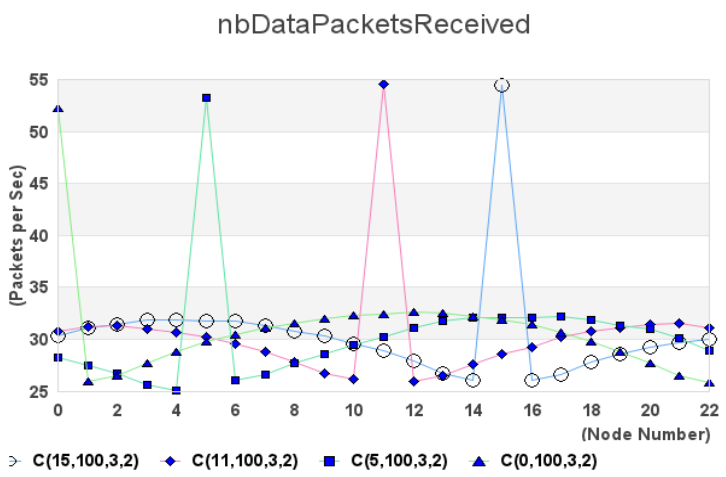

(b)

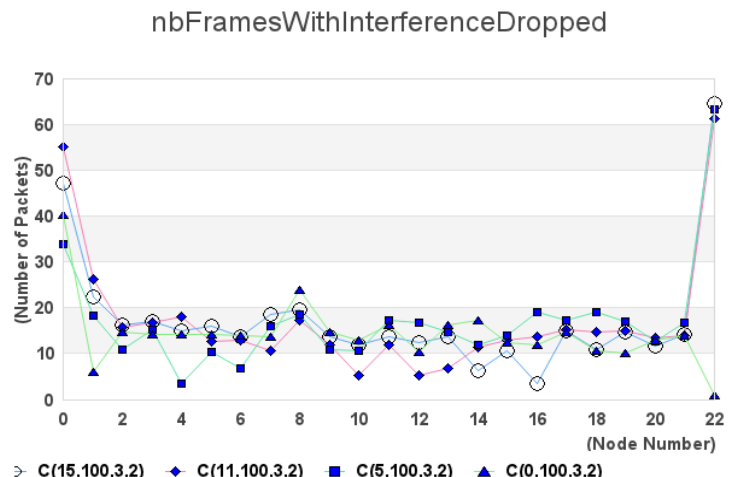

(c)

Figure 6. (a) Comparison of performance for different gateway locations for the circular path. (b) The number of packets received by routers. (c) Dropped packets for routers experiencing interference for the circular path. 
2) Circular paths: The length for the circular path was approximately $2.5 \mathrm{~km}$, and a total of 23 routers (including one gateway) were installed, as shown in Fig. 4(b). The circular path network forms a loop. Unlike that in a straight path, broadcasting packets are transmitted within a loop. Figure 6(a) is a table of four gateway location configurations. The simulation results for the five performance factors of the four configurations did not differ significantly. However, regarding the packets discarded or dropped because of node interference (Fig. 6(c)), the number of packets dropped for Routers 0, 1, 21, and 22 was approximately two to three times that of the other nodes. Regarding the routers located on the left-end of the path (Routers 0 and 22) shown in Fig. 4(b), the larger turning angles of this path cause greater overlapping of the router regions or domains, resulting in more wireless signal interference and a higher packet drop probability.

\section{CONCLUSION}

In this study, we used the green power and WSN techniques to address issues related to cycling activities, including health records, trajectory monitoring, group positioning, and immediate safety issues. We designed a path establishment simulation system to enable managers to perform path simulations before the establishment of physical paths. Paths are simulated using data of bicycle traffic and path shapes to determine the optimum establishment of routers and gateways. Following simulation, establishment reports detailing the latitude and longitude of all routers and gateways are used by construction personnel to complete the installation of sensor networks. The designs and service frameworks proposed in this study can be employed for large-scale establishment and installation, such as in national forest parks, to enable park management to control the location and safety of tourists easily.

\section{ACKNOWLEDGMENT}

This work was supported by the Ministry of Science and Technology, R.O.C., under grant 104-2745-E-019-002

\section{REFERENCES}

[1] V. Jelicic, M. Magno, G. Paci, D. Brunelli and L. Benini, "Design, characterization and management of awireless sensor network for smart gas monitoring", 4th IEEE International Workshop on Advances in Sensors and Interfaces (IWASI), pp. 115-120, Brindisi, Italy, 2011.
[2] L.W. Yeh, C.Y. Lu, C.W. Kou, Y.C. Tseng and C.W. Yi, "Autonomous Light Control by Wireless Sensor and Actuator Networks", IEEE Sensors Journal, vol. 10, no. 6, June 2010.

[3] P.Y. Chen, W.T. Chen, Y.C. Tseng and C.F. Huang, "Providing Group Tour Guide by RFIDs and Wireless Sensor Networks", IEEE Trans. On Wireless Communications, vol. 8, no. 6, June 2009.

[4] R.S. Dilmaghani, H. Bobarshad, M. Ghavami, S. Choobkar and C. Wolfe, "Wireless Sensor Networks for Monitoring Physiological Signals of Multiple Patients", IEEE Trans. on Biomedical Circuits and Systems, vol. 5 , no. 4, pp. 347-356, 2011.

[5] S.R. Huang, G.J. Horng and G.J. Jong, "Intelligent Hospital Space Platform Combined with RFID and Wireless Sensor Network", IEEE International Conference on Intelligent Information Hiding and Multimedia Signal Processing, pp. 1001-1004, Harbin, China, 2008.

[6] J. Guevara, F. Barrero, E. Vargas, J. Becerra and S. Toral, "Environmental wireless sensor network for road traffic applications", IET Intelligent Transport Systems, vol. 6, issue 2, pp. 177-186, 2012.

[7] T. Naumowicz, R. Freeman, H. Kirk, B. Dean, M. Calsyn, A. Liers, A. Braendle, T. Guilford and J. Schiller, "Wireless Sensor Network for Habitat Monitoring on Skomer Island", 5th IEEE International Workshop on Practical Issues in Building Sensor Network Applications, pp. 882-889, Denver, Colorado, 2010.

[8] A.K. Othman, K.M. Lee, H. Zen, W.A.Wan Zainal and M.F. M. Sabri, "Wireless Sensor Networks for Swift Bird Farms Monitoring", IEEE International Conference on Ultra Modern Telecommunications \& Workshops, pp. 1-7, Petersburg, Russia, 2009.

[9] K. Chintalapudi, T. Fu, J. Paek, N. Kothari, S. Rangwala, J. Caffrey, R. Govindan, E. Johnson and S. Masri, "Monitoring Civil Structures with a Wireless Sensor Network", IEEE Internet Computing, vol. 10, issue 2, pp. 26-34, 2006.

[10] C. Xiaojuan, W. Dargie and L. Guan, "Energy Model for H2S Monitoring Wireless Sensor Network", 11th IEEE International Conference on Computational Science and Engineering, pp. 402-409, SP, Brazil, 2008.

[11] H. Matsuo, W. Lin, F. Kurokawa, T. Shigemizu and N. Watanabe, "Characteristics of the Multiple-Input DC-DC Converter," IEEE Trans. on Industrial Electronics, vol. 51, no. 3, pp. 625-631, 2004

[12] J. Y. June and J. W. Lee, "ZigBee Device Access Control and Reliable Data Transmission in ZigBee Based Health Monitoring System", International Conference on Advanced Communication Technology. ICACT'08, Gangwon-Do, pp. 795-797, 2008.

[13] F. Gustafsson, F. Gunnarsson, N. Bergman, U. Forssell, J. Jansson and R. Karlsson, "Particle filters for positioning, navigation, and tracking," IEEE Trans. on signal processing, vol. 50, no. 2, pp. 425-438, 2002.

[14] See, C.H., Horoshenkov, K. V., Abd-Alhameed, R.A., Yim Fun Hu, Tait, S. J., "A Low Power Wireless Sensor Network for Gully Pot Monitoring in Urban Catchments", IEEE Sensors Journal, vol. 12, no. 5, pp. 15451553, May. 2012.

[15] Young-Huk Ha, "Dynamic integration of zigbee home networks into home gateways using OSGI service registry", IEEE Trans. Consumer Electronics, vol. 40, no. 2, pp. 470-476, May. 2009.

[16] OMNeT++, http://www.omnetpp.org/.

[17] MiXiM, http://mixim.sourceforge.net/. 